\title{
MS08-P121 LATE | The ESCRT-III Protein Vps24 Forms Double Stranded Filaments Composed Of Domain-Swapped Dimers
}

Huber, Stefan (TU Delft, Delft); Mortensen, Simon A. (Forschungszentrum Jülich GmbH, Juelich, GER); Sachse, Carsten (Forschungszentrum Jülich GmbH, Juelich, GER)

ESCRT-III proteins mediate a range of membrane remodeling events such as multivesicular body biogenesis, cytokinesis and viral budding. Little atomic resolution information is available on how ESCRT-III monomers come together to facilitate membrane remodeling. In this study we determined the cryo-EM structure of a filamentous assembly of yeast Vps24 at $3.2 \AA$ resolution. We found that Vps24 monomers adopt an elongated open conformation and bind together with another monomer to form a domain-swapped dimer with a central symmetry axis. The dimers combine to form an apolar ribbon, and two of those form a double stranded filament. The observation of such an architecture is unexpected in the context of ESCRT-III assemblies and strikingly different from proposed linear Snf7 filaments. Our structure of homopolymeric Vps24 suggests a new architecture on how Vps24 may assemble in hetero-polymers as they form in the cell during membrane remodelling events. 